\title{
Utilidad del índice de choque para predecir la mortalidad en pacientes pediátricos con sepsis grave o choque séptico
}

\author{
Claudia S. López-Reyes ${ }^{1}$, Lilia N. Baca-Velázquez¹, Miguel A. Villasis-Keever ${ }^{2}$ y Jessie N. Zurita-Cruz ${ }^{3 *}$ \\ ${ }^{1}$ Unidad de Terapia Intensiva Pediátrica; ${ }^{2}$ Unidad de Investigación Médica en Epidemiología Clínica; ${ }^{3}$ Unidad de Investigación en Nutrición. Unidad \\ Médica de Alta Especialidad, Hospital de Pediatría Dr. Silvestre Frenk Freund, Centro Médico Nacional Siglo XXI, Ciudad de México, México
}

\begin{abstract}
Resumen
Introducción: La sepsis en pediatría es la principal causa de muerte hospitalaria. Se han intentado crear herramientas que faciliten su identificación, como el índice de choque (IC), definido como el cociente entre frecuencia cardíaca y tensión arterial sistólica. El objetivo de este trabajo fue identificar el mejor momento del IC para predecir la mortalidad en pacientes pediátricos con sepsis grave y choque séptico. Métodos: Cohorte retrospectiva con 165 pacientes pediátricos que desarrollaron sepsis grave y choque séptico en la Unidad de Terapia Intensiva Pediatrica. Se calculó el IC al diagnóstico (ICO), a las dos, cuatro y seis horas posteriores al mismo (IC2, IC4 e IC6). Se crearon dos grupos según el desenlace (sobrevivientes/muertos). La comparación estadística se realizó con prueba U-Mann Whitney y $\chi^{2}$. Se compararon los factores de riesgo entre los sobrevivientes y muertos, calculando la razón de momios (RM). Resultados: Se comparó el valor del IC entre grupos; en el grupo de choque séptico, el IC se encontró más elevado en IC4 e IC6 ( $p=0.010$ y $p=0.005)$. Se encontró que, en los pacientes muertos, el IC incrementó progresivamente su valor en IC4 e IC6 $(p<0.05)$. Los valores de IC4 e IC6 elevados incrementaron el riesgo de muerte en los pacientes (IC4, RM: 442.1; IC 95\% [intervalo de confianza al 95\%]: 54.2-3,601.7; $p$ < 0.001; e IC6, RM: 81,951.3; IC 95\%: 427.1-15,700,000; $p$ < 0.001). Conclusiones: La elevación del valor del IC se asocia con mayor mortalidad de la sepsis. El valor de IC6 es el más útil para predecir mortalidad.
\end{abstract}

Palabras claves: Sepsis grave. Choque séptico. Índice de choque.

\section{Shock index utility to predict mortality in pediatric patients with septic shock or severe sepsis}

\begin{abstract}
Background: Pediatric sepsis is considered the main cause of hospital death around the world. Many groups have tried to create tools that facilitate its early identification, as the shock index (SI) defined as the ratio between cardiac frequency and systolic blood pressure. The objective of this study was to determine the utility of SI to predict mortality in pediatric patients with severe sepsis and septic shock. Methods: Retrospective cohort with 165 pediatric patients with severe sepsis or septic shock in the Pediatric Intensive Care Unit. SI was calculated at diagnosis, 2, 4 and 6 hours after (SI2, S/4 and S/6). We divided the population in two groups depending their outcome: survivors and non-survivors. The statistical analysis was
\end{abstract}


performed with U Mann-Whitney and chi squared tests. The risk factors were compared between the survivors and the dead, and we calculated the odds ratio (OR). Results: The median value of SI was compared between groups; in the group of septic shock, SI showed a tendency to remain high in SI4 and SI6 ( $p=0.010$ and $p=0.005$, respectively). Among the survivors and the non-survivors, we found that in the latter, $S I$ was progressively increased in SI4 and SI6 $(p<0.05)$. High values of S/4 and S/6 increased the risk of death in patients (SI4: OR: 442.1; CI 95\% [confidence interval 95\%]: 54.2-3,601.7; $p<0.001$ and SI6: OR: 81,951.3; Cl 95\%: 427.1-15,700,000]; $p<0.001$ ). Conclusions: High values of SI are associated with increased mortality. The IS6 value is the most useful to predict mortality.

Key words: Severe sepsis. Septic shock. Shock index.

\section{Introducción}

La sepsis en el niño es un importante problema de salud, ya que se considera la principal causa de muerte hospitalaria a nivel mundial. Se ha reportado que el $8.2 \%$ de los niños ingresados en Unidades de Terapia Intensiva Pediátrica (UTIP) presentan sepsis: el 4\% sepsis grave y el $2 \%$ choque séptico con una mortalidad del 25 al $48 \%$, dependiendo de las comorbilidades del paciente ${ }^{1-5}$.

El diagnóstico de sepsis se realiza de acuerdo con datos clínicos, entre los que se encuentran la frecuencia cardíaca $(\mathrm{FC})$, la temperatura y la frecuencia respiratoria; sin embargo, estos pueden mezclarse con datos de respuesta al trauma, que necesariamente son datos de un proceso infeccioso. Ante esto, se han estudiado múltiples marcadores bioquímicos que apoyan la identificación de la presencia, gravedad y pronóstico de la sepsis ${ }^{6}$. El marcador más utilizado en la práctica médica es el lactato sérico; sin embargo, no siempre está disponible en algunas unidades o simplemente no se realiza de forma oportuna, ya que los hallazgos clínicos no justifican la medición sistemática del mismo, lo cual retrasa el diagnóstico y tratamiento de los pacientes graves ${ }^{7}$.

Existen numerosas escalas pronósticas y diagnósticas para clasificar pacientes con sepsis grave o choque séptico, que valoran grados de afección fisiológica y cuyo sinergismo mejora la detección de las alteraciones hemodinámicas presentes. Sin embargo, la gran cantidad de datos a analizar (como la toma aislada de signos vitales, el monitoreo invasivo de presión venosa central, tensión arterial aislada, determinación de gases sanguíneos, entre otros) presentan inconvenientes como la seguridad de los resultados, el tiempo, los recursos y los costos necesarios para su realización, lo que complica en extremo estas determinaciones, y las convierte en demasiado complejas para su utilización en la práctica clínica ${ }^{8}$.

Se ha observado en diversos estudios que el índice de choque (IC), definido como el cociente entre la FC y la presión arterial sistólica (TAS), puede predecir de forma temprana la hipoxia celular y el compromiso hemodinámico ${ }^{9-11}$. Esto ha apoyado su uso como un marcador de detección temprana de sepsis, y se ha demostrado que los pacientes con un IC $>0.7$ presentaron tres veces más hiperlactatemia que aquellos con un IC $<0.7^{12}$.

Se ha analizado la asociación del IC como un indicador temprano de sepsis en la población pediátrica, aunque los estudios descritos no determinan diferentes puntos de corte por grupo etario, el cual, por condiciones fisiológicas, no es el mismo en toda la población pediátrica. Por ello, se decidió realizar este estudio con el objetivo de identificar el mejor momento para la medición del IC en la población pediátrica para predecir la mortalidad en sepsis grave o choque séptico.

\section{Métodos}

Se realizó una cohorte retrospectiva del periodo de enero del 2012 a abril 2014, en la que se incluyeron pacientes de entre un mes de edad y 16 años y 9 meses que desarrollaron sepsis grave o choque séptico durante su estancia en la UTIP. No fueron incluidos pacientes que fallecieron dentro de las primeras seis horas después del ingreso en la UTIP. El tiempo cero se definió como el momento de ingreso del paciente con sospecha de sepsis en la UTIP.

De acuerdo con la valoración que realizaron el médico tratante y los investigadores acerca de los datos clínicos y de laboratorio, los pacientes se clasificaron conforme a los criterios establecidos por la Internacional Pediatric Sepsis Consensus Conference 2005 con evento de sepsis grave o choque séptico ${ }^{6}$.

A partir de los registros de enfermería, se identificaron los valores de la FC y TAS en el momento del diagnóstico de sepsis grave o choque séptico, y a las dos, cuatro y seis horas posteriores. Se obtuvo el cociente entre FC y TAS para obtener el valor de IC correspondiente a cada momento. 
Tomando las mediciones de FC y TAS desde el momento de la sospecha de sepsis grave o choque séptico, y consecutivamente cada dos horas durante las primeras seis horas, se determinó el IC para cada sujeto en cada momento de medición para construir cuatro momentos de análisis en el tiempo (IC al diagnóstico, a las dos, cuatro y seis horas posteriores: ICO, IC2, IC4 e IC6), y se realizó una comparación en dos grupos de acuerdo con el desenlace de los pacientes (sobrevivientes vs. fallecidos).

En ambos grupos se analizaron variables que podían modificar el desenlace, como la edad, sexo, inmunocompromiso, problemas cardíacos, días de estancia intrahospitalaria; y en la UTIP, uso de dobutamina, adrenalina, noradrenalina, levosimendán e hidrocortisona o variables que podían modificar el IC, como la presencia de problemas cardiovasculares y la edad.

Se consideraron como inmunocomprometidos a los pacientes hematoncológicos que hubieran recibido quimioterapia u otra condición en la que estuvieran indicadas dosis altas de esteroides que condicionaran inmunosupresión. Se definió como problema cardíaco a pacientes con cardiopatías congénitas o adquiridas.

Antes del inicio del estudio, el protocolo fue aprobado por el Comité de Investigación y Ética en Salud del Hospital de Pediatría Dr. Silvestre Frenk Freund.

\section{Análisis estadístico}

Se utilizaron medidas de tendencia central y de dispersión de acuerdo con la escala de medición de las variables. Se aplicó la prueba de Shapiro-Wilks, para determinar las variables cuantitativas que tuvieron una distribución diferente a la normal.

Para comparar las variables cuantitativas entre los grupos, se aplicó la prueba de U-Mann Whitney, y para variables cualitativas, la prueba de $\chi^{2}$. Se compararon los factores de riesgo entre los pacientes vivos y los fallecidos, calculando la razón de momios (RM) y sus intervalos de confianza. Se construyeron dos modelos de regresión logistica múltiple para el IC al tiempo 4 y al tiempo 6. En estos dos modelos, se incluyeron además las variables que en análisis bivariado mostraron un valor $p<0.1$.

Todos los análisis se realizaron con el software SPSS versión 17.0 (IBM).

\section{Resultados}

En el estudio se incluyeron 165 pacientes que ingresaron en terapia intensiva en el periodo establecido. Sin
Tabla 1. Características infecciosas y aislamiento de microorganismos de los pacientes incluidos en el estudio

\begin{tabular}{|l|c|}
\hline Características & n (\%) \\
\hline Sexo & \\
\hline Masculino & $82(49.7)$ \\
\hline Femenino & $83(50.3)$ \\
\hline Grupo de edad & \\
\hline Lactante & $76(46.1)$ \\
\hline Preescolar & $27(16.4)$ \\
\hline Escolar & $37(22.4)$ \\
\hline Adolescente & $25(15.2)$ \\
\hline Padecimiento de base & \\
\hline Oncológicos & $33(20)$ \\
\hline Cardiovasculares & $30(18.2)$ \\
\hline Gastrointestinales & $30(18.2)$ \\
\hline Neurológicos & $25(15.2)$ \\
\hline Hematológicas & $12(7.3)$ \\
\hline Sano previamente & $12(7.3)$ \\
\hline Genopatías & $8(4.8)$ \\
\hline Inmunológicas & $8(4.8)$ \\
\hline Respiratorias & $3(1.8)$ \\
\hline Endocrinológicas & $2(1.2)$ \\
\hline Renales & $2(1.2)$ \\
\hline Motivo de ingreso en la UTIP & $37(22.4)$ \\
\hline Neumonía adquirida en la comunidad & $32(19.4)$ \\
\hline Complicaciones oncológicas & $21(12.7)$ \\
\hline Erisis convulsivas/Estado epiléptico & $13(7.9)$ \\
\hline Sepsis abdominal & $11(6.7)$ \\
\hline Neumonía intrahospitalaria & $10(6.1)$ \\
\hline Otros* & $41(24.8)$ \\
\hline
\end{tabular}

*Falla cardíaca $(6,3.6 \%)$; neuroinfección $(4,2.4 \%)$; hemorragia intracraneal $(4,2.4 \%)$; sepsis $(3,1.8 \%)$; polirradiculoneuropatías $(3,1.8 \%)$; derrame pleural $(3,1.8 \%)$; sangrado de tubo digestivo $(3,1.8 \%)$; encefalopatía hepática $(3,1.8 \%)$; broncoespasmo grave $(2,1.2 \%)$; edema pulmonar agudo $(2,1.2 \%)$; crisis de hipoxia $(2,1.2 \%)$; bloqueo auriculoventricular $(1,0.6 \%)$; choque hipovolémico $(1,0.6 \%)$; infección quirúrgica $(1,0.6 \%)$; cetoacidosis diabética $(1,0.6 \%)$; traqueítis bacteriana $(1,0.6 \%)$; gastroenteritis infecciosas $(1,0.6 \%)$.

UTIP: Unidad de Terapia Intensiva Pediátrica

existir predominio de sexo, con respecto al grupo etario, el más frecuente fue el de los lactantes $(46.1 \%, n=76)$. Los padecimientos de base más frecuentes fueron los oncológicos, cardiovasculares y gastrointestinales $(56.4 \%, n=93)$ y solamente el $7.3 \%(n=12)$ eran pacientes previamente sanos. La neumonía adquirida en la comunidad y las complicaciones oncológicas fueron las patologías más frecuentes que condicionaron el ingreso a la UTIP en el $41.8 \%(n=69)$ (Tabla 1$)$.

De los 165 pacientes incluidos, 137 (83\%) cumplieron criterios de choque séptico; el resto presentaba sepsis grave. El origen de la sepsis se identificó como foco pulmonar en el $49.6 \%$ de los pacientes; en menos de la mitad de los casos se logró aislar algún microorganismo a través de hemocultivos, cultivo de catéter venoso central, de secreción bronquial o urocultivo. E 
microorganismo que más frecuentemente se aisló fue Klebsiella pneumoniae en el $5.5 \%$ de los pacientes (Tabla 2). Se presentaron 69 defunciones (41.8\%) atribuidas a choque séptico.

Al comparar algunas características entre los pacientes sobrevivientes y los fallecidos, se observaron diferencias estadísticas en el uso de adrenalina, levosimendán e hidrocortisona y la presencia de choque séptico. De los pacientes sobrevivientes, el $70.8 \%$ presentaron choque séptico, mientras que del grupo que fallecieron, lo presentaron el $100 \%$ (Tabla 3).

Al realizar el análisis de acuerdo con la condición del paciente, el valor de la mediana en los momentos ICO, IC2, IC4 e IC6 fue de 2.1, 2.0, 1.6 y 1.4, respectivamente, en el grupo de sepsis grave. En el grupo de choque séptico, en los momentos IC0, IC2, IC4 e IC6, la mediana fue de 2.0, 2.0, 1.9 y 1.7, respectivamente.

$\mathrm{Al}$ analizar el comportamiento del IC entre los pacientes que sobrevivieron y los que murieron, se determinó que los valores de la mediana en los momentos IC0, IC2, IC4 e IC6 fueron 2.0, 2.0, 1.6 y 1.3 en el grupo de sobrevivientes, mientras que los valores fueron de 2.0, 2.1, 2.2 y 2.4, respectivamente, para aquellos que fallecieron. Como se observa en la tabla 3, la mediana de ICO fue similar entre los dos grupos $(p=0.642)$. Sin embargo, en IC2, IC4 e IC6 se puede observar que el valor tiende a disminuir en los sobrevivientes, mientras que en los pacientes que finalmente fallecieron, el valor va incrementando, lo cual fue estadísticamente significativo (IC2: $p=0.045$; IC4: $p=0.000$, e IC6: $p=0.000$ ) (Tabla 3). Específicamente, de los 38 pacientes que a las cuatro horas de seguimiento tenían un IC $\geq 2.2$, solo cuatro sobrevivieron, mientras que de los 37 pacientes que a las seis horas de seguimiento tuvieron un $I C \geq 2.4$, ninguno sobrevivió.

Se calculó la RM del IC en cada tiempo para predecir el desenlace (muerte). Para ICO e IC2, no se encontró significación estadística (IC0: RM: 0.7; IC 95\% [intervalo de confianza al 95\%]: 0.32-1.5; $p=0.42$ e IC2: RM: 2.16; IC 95\%: 0.9-4.8; $p=0.6$ ), mientras que para IC4 e IC6, sí se encontró significación estadística (IC4: RM: 18.9; IC 95\%: 7.2-49.6; $p<0.001$ e IC6: RM: 72.6; IC 95\%: 21.3-246.9; $p<0.001$ ).

En los modelos de regresión logística múltiple, además de IC4 e IC6, se incluyeron el sexo, la edad (meses), inmunocompromiso, problemas cardíacos, días de estancia intrahospitalaria y en la UTIP, además del uso de vasoactivos. En el primer modelo realizado para IC4, se pudo observar una asociación positiva del IC (RM: 442.1; $p$ < 0.001) con la muerte del paciente en forma independiente. En este mismo modelo se observó que,
Tabla 2. Características infecciosas y aislamiento de microorganismos de los pacientes incluidos en el estudio

\begin{tabular}{|c|c|}
\hline Características & n (\%) \\
\hline $\begin{array}{l}\text { Gravedad de la sepsis } \\
\text { Choque séptico } \\
\text { Sepsis grave }\end{array}$ & $\begin{array}{c}137(83) \\
28(17)\end{array}$ \\
\hline $\begin{array}{l}\text { Foco infeccioso } \\
\text { Neumonía intrahospitalaria } \\
\text { Neumonía asociada con ventilador } \\
\text { Neumonía adquirida en la comunidad } \\
\text { Bacteriemia } \\
\text { Sepsis abdominal } \\
\text { Asociado con catéter venoso central } \\
\text { Neuroinfección } \\
\text { Absceso perirrenal }\end{array}$ & $\begin{array}{l}122(73) \\
33(20) \\
28(16.9) \\
21(12.7) \\
14(8.5) \\
13(7.8) \\
10(6.1) \\
2(1.2) \\
1(0.6)\end{array}$ \\
\hline $\begin{array}{l}\text { Cultivos con desarrollo } \\
\text { microbiológico } \\
\text { Hemocultivos periféricos } \\
\text { Cultivo de catéter venoso central } \\
\text { Cultivo de secreción bronquial } \\
\text { Urocultivo }\end{array}$ & $\begin{array}{c}42(25.4) \\
23(13.9) \\
10(6.0) \\
8(4.8) \\
1(0.6)\end{array}$ \\
\hline $\begin{array}{l}\text { Aislamiento microbiológico } \\
\text { Klebsiella pneumoniae } \\
\text { Pseudomonas aeruginosa } \\
\text { Escherichia coli } \\
\text { Enterobacter cloacae } \\
\text { Staphylococcus aureus } \\
\text { Otros* }\end{array}$ & $\begin{array}{l}42(25.4) \\
9(5.5) \\
8(4.8) \\
7(4.2) \\
5(3.0) \\
4(2.4) \\
9(5.5)\end{array}$ \\
\hline
\end{tabular}

*Aislamiento microbiológico: Streptococcus pneumoniae (2, 1.2\%); Acinetobacter baumani $(2,1.2 \%)$; Staphylococcus epidermidis $(1,0.6 \%)$; Burkholderia cepacia $(1,0.6 \%)$; Enterococcus fecalis $(1,0.6 \%)$; Candida parapsilosis $(1,0.6 \%)$ y Candida flamata $(1,0.6 \%)$.

de manera independiente, la edad (RM: 1.02; $p=0.001$ ), el uso de adrenalina (RM: $17.1 ; p=0.015)$ y noradrenalina (RM: $24.1 ; p=0.007)$ aumentó el riesgo de muerte en los pacientes. En el segundo modelo realizado para el IC6, se observó que el IC aumentó el riesgo de muerte en forma considerable, con RM: 81,951.3; $p<0.001$. También la edad aumentó el riesgo de muerte (RM: 1.04; $p<0.001)$ (Tabla 4).

\section{Discusión}

A pesar de los importantes avances en el manejo de la sepsis en pediatría, aún existe una alta morbilidad y mortalidad secundaria a sepsis grave y choque séptico. Por esta razón, es necesario contar con herramientas clínicas que ayuden a la detección temprana de estas condiciones, para poder implementar medidas terapéuticas que modifiquen su pronóstico. Dada la sencillez de su determinación, el IC puede ser difundido para su uso en el área de urgencias, donde la toma de signos 
Tabla 3. Comparación de las características entre los pacientes vivos y los que fallecieron

\begin{tabular}{|c|c|c|c|}
\hline \multirow[t]{2}{*}{ Características } & $\begin{array}{l}\text { Vivos } \\
(\mathrm{n}=96)\end{array}$ & $\begin{array}{l}\text { Fallecidos } \\
\qquad(n=69)\end{array}$ & \multirow[t]{2}{*}{$\mathbf{p}$} \\
\hline & \multicolumn{2}{|c|}{ n (\%) } & \\
\hline Sexo masculino & $48(50)$ & $34(49.2)$ & NS \\
\hline Edad (meses) & $31.5(1-199)$ & $29(1-199)$ & NS \\
\hline Inmunocomprometidos & $31(32.2)$ & $25(36.2)$ & NS \\
\hline Problema cardíaco & 18 (18.75) & $9(13.04)$ & NS \\
\hline Días de estancia intrahospitalaria & $8.5(0-124)$ & $11(1-94)$ & NS \\
\hline Días de estancia en la UTIP & $5(1-60)$ & $5(1-94)$ & NS \\
\hline Sepsis grave & $28(29.1)$ & - & 0.000 \\
\hline Choque séptico & $68(70.8)$ & $69(100)$ & \\
\hline $\begin{array}{l}\text { Uso } \\
\text { Dobutamina } \\
\text { Adrenalina } \\
\text { Noradrenalina } \\
\text { Levosimendán } \\
\text { Hidrocortisona }\end{array}$ & $\begin{array}{c}36(37.5) \\
34(35.4) \\
29(30.2) \\
5(5.2) \\
3(3.1)\end{array}$ & $\begin{array}{l}35(50.7) \\
44(63.7) \\
27(39.1) \\
12(17.3) \\
11(15.9)\end{array}$ & $\begin{array}{c}\text { NS } \\
0.000 \\
\text { NS } \\
0.01 \\
0.004\end{array}$ \\
\hline $\begin{array}{l}\text { Índice de choque } \\
0 \\
2 \\
4 \\
6\end{array}$ & $\begin{array}{c}2(1.1-2.6) \\
2(0.9-2.6) \\
1.6(0.9-2.5) \\
1.3(0.5-2.3)\end{array}$ & $\begin{array}{c}2(0.9-2.8) \\
2.1(1.1-3.2) \\
2.2(0.9-3.2) \\
2.4(0.8-3.2)\end{array}$ & $\begin{array}{c}\text { NS } \\
0.04 \\
0.000 \\
0.000\end{array}$ \\
\hline
\end{tabular}

NS: no significativo; UTIP: Unidad de Terapia Intensiva Pediátrica

vitales es una medida rutinaria, y no así la toma de otros marcadores tempranos de infección $n^{13,14}$.

Este estudio demostró que la elevación en el valor del IC se asocia directamente con una mayor mortalidad, y que un IC elevado a las cuatro y seis horas a partir del diagnóstico de sepsis grave o choque séptico predice un desenlace fatal del paciente; específicamente, el valor IC6 predice el fallecimiento del paciente (el $100 \%$ de los sujetos con IC6 $\geq 2.4$ fallecieron).

En niños, los valores normales del IC y su papel potencial para diagnosticar sepsis grave o choque séptico no han sido bien estudiados, pero algunos estudios han realizado mediciones del IC a las $0,1,2$, 4 y 6 horas después del ingreso y demostraron que, si a las 6 horas este valor disminuía, no se asociaba con la mortalidad; si se mantenía elevado, sí estaba asociado con un incremento en la mortalidad $(p=0.02$ vs. 0.03 ). Estos datos concuerdan con lo descrito en la literatura. Entre los estudios más significativos se encuentra el de Berger, et al., un estudio retrospectivo con una población de 146 pacientes, en el que se demostró que el IC era predictivo para el riesgo de muerte a las 0,4 y 6 horas después del diagnóstico con sepsis grave y choque séptico, y que los niños con un IC anormal, ajustado para la edad, a las 0 y 6 horas del diagnóstico, presentaban un riesgo relativo de muerte mayor que los niños con un IC normal, ajustado a la edad, a las 0 y 6 horas después del diagnóstico. En este estudio, además, se realizó una correlación con el lactato, marcador ampliamente utilizado para sepsis grave. Se demostró que el IC es un mejor marcador que la FC o la presión sistólica por separado. Este estudio no dividió a los pacientes por grupo etario y los autores perdieron la correlación inicial con el lactato, lo que les pudo haber dado una mayor información ${ }^{15}$.

Yasaka, et al. realizaron un estudio retrospectivo, y observaron que el IC al ingreso y hasta seis horas posteriores al diagnóstico estaba asociado con la mortalidad al ingreso a UTIP. Al realizar la estratificación, observaron que el IC solamente se asoció con la mortalidad en niños de 1-3 años $y \geq 12$ años ${ }^{17}$. En ciertos grupos, la mejora en el IC en la UTIP respecto a su 
Tabla 4. Regresión logística múltiple para calcular el riesgo del índice de choque a las cuatro y seis horas de seguimiento para presentar muerte en pacientes pediátricos con sepsis grave y choque séptico

\begin{tabular}{|c|c|c|c|}
\hline Características analizadas & $\mathbf{R M}$ & IC $95 \%$ & $\mathbf{p}$ \\
\hline Indice de choque (a las 4 horas) & 442.1 & $54.2-3,601.7$ & $<0.001$ \\
\hline Sexo & 0.59 & $0.21-1.61$ & NS \\
\hline Edad (meses) & 1.02 & $1.009-1.03$ & 0.001 \\
\hline Inmunocomprometidos & 2.6 & $0.7-9.6$ & NS \\
\hline Problema cardíaco & 0.41 & $0.1-1.7$ & NS \\
\hline Días de estancia intrahospitalaria & 0.97 & $0.9-1.02$ & NS \\
\hline Días de estancia en la UTIP & 1.08 & $0.9-1.1$ & NS \\
\hline $\begin{array}{l}\text { Uso } \\
\text { Dobutamina } \\
\text { Adrenalina } \\
\text { Noradrenalina } \\
\text { Levosimendán } \\
\text { Hidrocortisona }\end{array}$ & $\begin{array}{c}0.78 \\
17.1 \\
24.1 \\
1.6 \\
1.3\end{array}$ & $\begin{array}{c}0.2-2.2 \\
1.7-168.9 \\
2.4-239.2 \\
0.2-9.7 \\
0.2-9.3\end{array}$ & $\begin{array}{l}\text { NS } \\
0.015 \\
0.007 \\
\text { NS } \\
\text { NS }\end{array}$ \\
\hline Indice de choque (a las 6 horas) & $81,951.3$ & $427.1-15,700,000$ & $<0.001$ \\
\hline Sexo & 0.1 & $0.02-1.4$ & NS \\
\hline Edad (meses) & 1.04 & $1.02-1.07$ & $<0.001$ \\
\hline Inmunocomprometidos & 5.01 & $0.3-82.0$ & NS \\
\hline Problema cardíaco & 0.3 & $0.02-3.6$ & NS \\
\hline Días de estancia intrahospitalaria & 0.9 & $0.7-1.1$ & NS \\
\hline Días de estancia en la UTIP & 1.2 & $0.9-1.6$ & NS \\
\hline $\begin{array}{l}\text { Uso } \\
\text { Dobutamina } \\
\text { Adrenalina } \\
\text { Noradrenalina } \\
\text { Levosimendán } \\
\text { Hidrocortisona }\end{array}$ & $\begin{array}{c}0.3 \\
46.2 \\
61.8 \\
0.17 \\
0.2\end{array}$ & $\begin{array}{c}0.03-3.6 \\
0.2-10,110 \\
0.2-15,625 \\
0.008-3.5 \\
0.01-6.0\end{array}$ & $\begin{array}{l}\text { NS } \\
\text { NS } \\
\text { NS } \\
\text { NS } \\
\text { NS }\end{array}$ \\
\hline
\end{tabular}

IC: intervalo de confianza; RM: razón de momios; UTIP: Unidad de Terapia Intensiva Pediátrica

admisión se asoció con una mejoría en la evolución respecto a los niños con un IC más alto a su ingreso. Estas observaciones demostraron que la determinación del IC en las primeras seis horas de admisión en la UTIP es una herramienta adicional para valorar la respuesta a la terapia de resucitación inicial.

Por otro lado, se debe aclarar que el resultado del análisis multivariado con respecto al aumento del riesgo de fallecer por el uso de adrenalina y levosimendán se estudió debido a que se ha observado que, en los sujetos que requieren el uso de estos medicamentos, generalmente persisten alteraciones hemodinámicas graves ${ }^{16}$.

Los resultados de este estudio concuerdan con los datos reportados en la literatura ${ }^{17-19}$. Las limitaciones del mismo son el tamaño de la muestra, la cual fue pequeña en comparación con otros estudios; la recolección de los datos, ya que al ser retrospectiva está limitada a la información documentada en los expedientes, y en algunas ocasiones es insuficiente. Por esta razón, se considera conveniente realizar en un futuro un estudio prospectivo que permita al investigador obtener de forma más fidedigna la información a analizar.

Se puede concluir que, en general, el mejor momento para predecir la mortalidad mediante el valor de IC es a las seis horas de iniciada la reanimación en pacientes pediátricos con sepsis grave o choque séptico, teniendo una RM: 81,951.3; IC 95\%: 427.1$15,700,000$. La difusión de la utilidad del IC en los pacientes pediátricos, sobre todo en los servicios de primer contacto, así como su determinación, pueden 
ser útiles para modificar la evolución dentro de las primeras seis horas de estancia en la UTIP de un paciente con sepsis o choque séptico.

\section{Conflicto de intereses}

Los autores declaran no tener ningún conflicto de intereses.

\section{Responsabilidades éticas}

Protección de personas y animales. Los autores declaran que los procedimientos seguidos se conformaron a las normas éticas del comité de experimentación humana responsable y de acuerdo con la Asociación Médica Mundial y la Declaración de Helsinki.

Confidencialidad de los datos. Los autores declaran que han seguido los protocolos de su centro de trabajo sobre la publicación de datos de pacientes.

Derecho a la privacidad y consentimiento informado. Los autores han obtenido el consentimiento informado de los pacientes y/o sujetos referidos en el artículo. Este documento obra en poder del autor de correspondencia.

\section{Bibliografía}

1. Bryce J, Boschi Pinto C, Shibuya K, Black RE. WHO estimates of the causes of death in children. Lancet. 2005;365:1147-52.

2. Dellinger RP, Carlet JM, Masur H, Gerlach H, Calandra T, Cohen J, et al. Surviving Sepsis Campaign guidelines for management of severe sepsis and septic shock. Crit Care Med. 2004;32:858-73.

3. Hartman ME, Linde-Zwirble WT, Angus DC, Watson RS. Trends in the epidemiology of pediatric severe sepsis. Pediatr Crit Care Med. 2013;14 686-93.

4. Weiss SL, Fitzgerald JC, Pappachan J, Wheeler D, Jaramillo-Bustamante JC, Salloo A, et al. Sepsis Prevalence, Outcomes, and Therapies
(SPROUT) Study Investigators and Pediatric Acute Lung Injury and Sepsis Investigators (PALISI) Network. Global epidemiology of pediatric severe sepsis: the sepsis prevalence, outcomes, and therapies study. Am J Respir Crit Care Med. 2015;191:1147-57.

5. Stevenson EK, Rubenstein AR, Radin GT, Wiener RS, Walkey AJ. Two decades of mortality trends among patients with severe sepsis: a comparative meta-analysis. Crit Care Med. 2014;42:625-31.

6. Goldstein B, Giroir B, Randolph A, and the members of the International Consensus Conference on Pediatrics Sepsis. International pediatric sepsis consensus conference: Definitions for sepsis and organ dysfunction in pediatrics. Pediatr Crit Care Med. 2005;6:2-7.

7. Pacheco-Rosas DO, Huelgas-Plaza AC, Miranda-Novales MG. Serum lactate as a biomarker of severe sepsis in children with cancer, neutropenia and fever. Rev Med Inst Mex Seguro Soc. 2014;52:S24-9.

8. Alkhouli M, Solaiman AY, Zhao H, Morad A, Escarcega RO, O'Murchu B, et al. Clinical utility of central venous saturation for the calculation of cardiac index in cardiac patients. J Card Fail. 2014;20:716-22.

9. Zhang $X$, Wang $Z$, Wang Z, Fang M, Shu Z. The prognostic value of shock index for the outcomes of acute myocardial infarction patients: A systematic review and meta-analysis. Medicine (Baltimore). 2017; 96:e8014.

10. Kobayashi A, Misumida N, Luger D, Kanei Y. Shock Index as a predictor for in-hospital mortality in patients with non-ST-segment elevation myocardial infarction. Cardiovasc Revasc Med. 2016;17:225-8.

11. Strehlow MC. Early identification of shock in critically ill patients. Emerg Med Clin North Am. 2010;28:57-66.

12. Rousseaux J. Prognostic value of shock index in children with septic shock. Pediatr Emerg Care. 2013:29:1055-9.

13. Torabi M, Moeinaddini S, Mirafzal A, Rastegari A, Sadeghkhani N. Shock index, modified shock index, and age shock index for prediction of mortality in Emergency Severity Index level 3. Am J Emerg Med. 2016;34:2079-83.

14. Ray S, Cvetkovic M, Brierley J, Lutman DH, Pathan N, Ramnarayan P, et al. Shock index values and trends in pediatric sepsis: Predictors or therapeutic targets? A retrospective observational study. Shock. 2016;46: 279-86.

15. Berger T, Green J, Horeczko T, Hagar Y, Garg N, Suarez A, et al. Shock index and early recognition of sepsis in the Emergency Department: pilot study. West J Emerg Med. 2013;14:168-74.

16. Belletti A, Benedetto U, Biondi-Zoccai G, Leggieri C, Silvani P, Angelini GD, et al. The effect of vasoactive drugs on mortality in patients with severe sepsis and septic shock. A network meta-analysis of randomized trials. J Crit Care. 2017;37:91-8.

17. Yasaka Y, Khemani RG, Markovitz BP. Is shock index associated with outcome in children with sepsis/septic shock? Pediatr Crit Care Med. 2013;14:e372-9.

18. Rappaport LD, Deakyne S, Carcillo JA, McFann K, Sills MR. Age and sex specific normal values for shock index in National Health and Nutrition Examination Survey 1999-2008 for ages 8 years and older. Am J Emerg Med. 2013;31:838-42.

19. Yussof SJ, Zakria MI, Mohamed FL, Bujang MA. Value of shock index in prognosticating the short-term outcome of death for patients presenting with severe sepsis and septic shock in the emergency department. Med $\mathrm{J}$ Malasia. 2012;67:406-11. 